Rhode Island College

Digital Commons @ RIC

\title{
Feasibility of Remote Management of Uncomplicated Urinary Tract Infection: A Quality Improvement Project
}

Scott A. Clement

Rhode Island College

Follow this and additional works at: https://digitalcommons.ric.edu/etd

Part of the Nursing Commons

\section{Recommended Citation}

Clement, Scott A., "Feasibility of Remote Management of Uncomplicated Urinary Tract Infection: A Quality Improvement Project" (2017). Master's Theses, Dissertations, Graduate Research and Major Papers Overview. 186.

https://digitalcommons.ric.edu/etd/186

This Major Paper is brought to you for free and open access by the Master's Theses, Dissertations, Graduate Research and Major Papers at Digital Commons @ RIC. It has been accepted for inclusion in Master's Theses, Dissertations, Graduate Research and Major Papers Overview by an authorized administrator of Digital Commons @ RIC. For more information, please contact digitalcommons@ric.edu. 


\section{FEASIBILITY OF REMOTE MANAGEMENT OF UNCOMPLICATED URINARY}

TRACT INFECTION: A QUALITY IMPROVEMENT PROJECT

by

Scott A. Clement, BSN, RN

A Major Paper Submitted in Partial Fulfillment

of the Requirements for the Degree of

Master of Science in Nursing

in

The School of Nursing

Rhode Island College

2017 


\begin{abstract}
Uncomplicated cystitis is a common condition that many women encounter in their lifetimes. The subjective symptoms that patients provide to their clinician are a critical component in the providers' diagnosis. Traditionally, uncomplicated cystitis is diagnosed and treated during a face-to-face consultation in primary care offices, urgent care centers and emergency departments. Quite often, clinicians treat their patients based upon their initial presenting symptoms, regardless of the point of care testing and before urine culture results are available. The purpose of this project was to determine how often subjective symptoms of uncomplicated urinary tract infections (UTIs) are associated with treatment sensitive to subsequent urine cultures. A comprehensive literature review was performed. The ACE Star Model of Knowledge Transformation was utilized to guide the research process. Data collection took place at Concentra Urgent Care in Warwick, RI after agency approval and IRB review. Eighty-four medical records of premenopausal (ages 18-45) non-pregnant females treated for UTI between January 1, 2016 and July 1, 2016 were reviewed during a retrospective chart review. Thirty-seven participants met the inclusion criteria for uncomplicated cystitis and presented with subjective symptoms of dysuria, urinary frequency and urinary urgency. Findings were consistent with research performed by Barry et al. (2001) and Hooton (2012). Barry et al. concluded that $64 \%$ of patients were appropriately treated remotely based upon subjective symptoms. Hooton also concluded that $90 \%$ of patients could be treated with appropriate antibiotics based upon their subjective symptoms. This research validated that $85.29 \%$ of patients could have been treated appropriately based solely on their subjective symptoms of uncomplicated cystitis. Recommendations and implications for advanced practice nursing are discussed.
\end{abstract}




\section{Table of Contents}

Background/Statement of the Problem ............................................................... 1

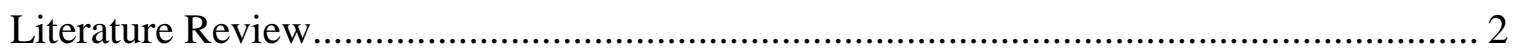

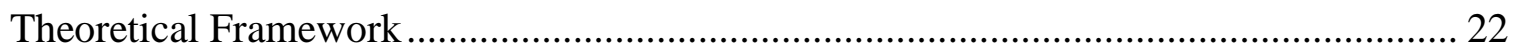

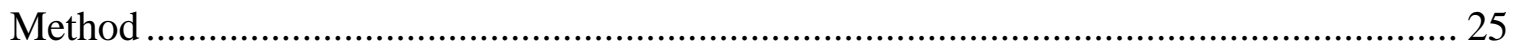

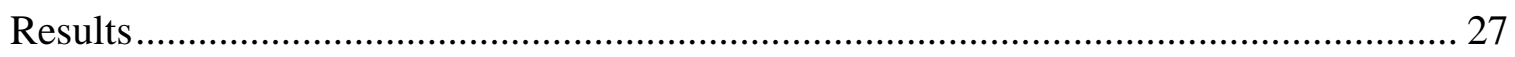

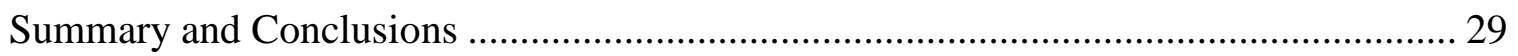

Recommendations and Implications for Advanced Nursing Practice .......................... 32

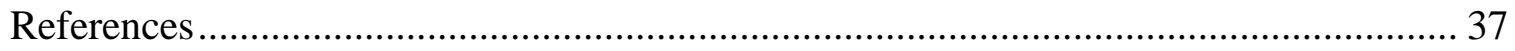

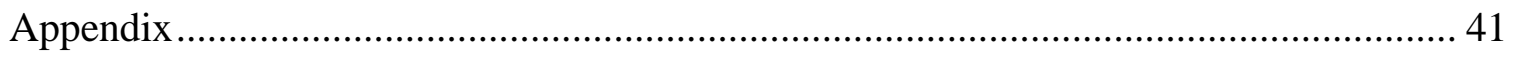




\section{FEASIBILITY OF REMOTE MANAGEMNT OF UNCOMPLICATED CYSTITIS}

\section{Background/Statement of the Problem}

Urinary tract infection (UTI) is the chief complaint in eight million clinic and emergency department visits, leading to approximately 100,000 hospital admissions each year (Dielubanza \& Schaeffer, 2011). According to the National Center for Health Statistics, pneumonia is the only infection treated more frequently than UTI in American emergency departments (NIH, 2013). The evaluation and treatment of community acquired UTI commands a significant portion of health care resources. Currently, the societal costs of these infections, including health care costs and time missed from work, are approximately $\$ 3.5$ billion per year in the United States alone (Flores-Mireles, Walker, Caparon, \& Hultgren, 2015). One in three women will develop a UTI requiring antibiotic treatment by age twenty-four and 50\% experience at least one UTI during their lifetime (Foxman, 2002).

Traditional UTI management requires the patient to seek treatment by face-to-face consultation with a medical provider, receive point of care testing and have their urine sample sent to a reference lab for culture and sensitivity. Research has proven that uncomplicated UTIs can be appropriately managed remotely with similar results as office consultations (Hooton, 2012). The purpose of this quality improvement project is to determine how often subjective symptoms of uncomplicated UTIs in an urgent care setting are associated with treatment sensitive to subsequent urine cultures.

Next, the review of literature will be presented. 


\section{Literature Review}

A search of the MEDLINE, PubMed, CINAHL, and Google Scholar was conducted using the terms urinary tract infections, cystitis, antibiotic prescribing, antibiotic resistance, prescribing guidelines, telemedicine, telephone management, and remote management. The search was conducted from 2007 to the present, including articles from 2001 in order to examine the literature over time related to acute uncomplicated cystitis management. The literature review will include the following subsections: epidemiology; urinary tract infections: definitions; pathophysiology; risk factors for urinary tract infections; diagnosing urinary tract infections and diagnostic testing; antimicrobial resistance; prescribing guidelines; and remote management strategies.

\section{Epidemiology}

Urinary tract infections are the most common bacterial infections in women, with one-half of women experiencing at least one UTI in their lifetime (Colgan \& Williams, 2011). It has been estimated that at least one-third of all women in the United States are diagnosed with a UTI by the time they reach 24 years of age (Foxman, 2014). Incidence and prevalence of UTI vary between different reports depending on the criteria used for diagnosis (urine culture vs. urinalysis) and the methods used for case ascertainment (Kattan \& Gordon, 2013). According to the National Health and Nutritional Examination Surveys (NHANES-III), the incidence is 53,067 per 100,000 adult women per year (Griebling, 2007). Most UTI's in women are acute uncomplicated cystitis caused by either Escherichia coli, Staphylococcus saprophyticus, Klebsiella species, Proteus species, Citrobacter species, or Enterococcus species (Colgan \& Williams). 
Antimicrobial agents used to treat UTIs account for nearly $15 \%$ of outpatient prescriptions (Dielubanza \& Schaeffer, 2011).

Although acute uncomplicated cystitis may not be thought of as a serious condition, patients' quality of life is often significantly affected (Colgan \& Williams, 2011). Acute uncomplicated cystitis results in an estimated six days of discomfort leading to approximately seven million office visits per year with an associated cost of $\$ 1.6$ billion (Foxman, 2014).

\section{Urinary Tract Infection: Definitions}

A urinary tract infection (UTI) is an infection in any part of the urinary system, including the kidneys, ureters, bladder, and urethra (Barber, Norton, Spivak, \& Mulvey, 2013). Urinary tract infections of the lower urinary tract are termed cystitis, which can be further classified as uncomplicated, complicated, or recurrent, depending on the symptoms and host characteristics. The focus on this literature review will concentrate on the most common form of UTI, acute uncomplicated cystitis. This is defined as the acute onset of painful urination (dysuria), with or without urinary frequency, urinary urgency, suprapubic pain, or hematuria in a healthy non-pregnant female with no known functional or anatomical abnormalities in the urinary tract (Grigoryan, Trautner, \& Gupta, 2014). Other characteristics in uncomplicated cystitis include that the host be immunocompetent, without comorbidities, and premenopausal (Colgan \& Williams, 2011). In addition to be classified as acute cystitis, the healthy female must present with initial or isolated symptoms (i.e. no previous $\mathrm{UTI} \leq 1$ yr.) (Dielubanza \& Schaeffer, 2011), no risk for sexually transmitted infection, vaginal discharge, or symptoms of upper urinary tract infection (pyelonephritis) (Grigoryan et al.). 
A complicated UTI, whether localized in the lower or upper urinary tract, is associated with an underlying condition that increases the risk of further related complications or failing antimicrobial therapy (Gupta, Hooton, \& Stamm, 2001). Host characteristics that define complicated cystitis include a history of childhood urinary tract infections, immunocompromised host, preadolescent or postmenopausal, pregnancy, underlying metabolic disorder (i.e. diabetes mellitus), and urologic abnormalities (e.g. stones, stents, indwelling catheters, neurogenic bladders, and polycystic kidney disease) (Colgan \& Williams, 2011). Complicated cystitis is initiated when the bacteria binds to urinary catheter, a kidney stone or bladder stone, or when retained in the urinary tract by a physical obstruction (Flores-Mireles et al., 2015).

Recurrent UTIs are defined as two uncomplicated infections in a six-month time period or three infections within a year (Dielubanza \& Schaeffer, 2011) or may be defined as urinary symptoms that persist or recur within a week or two of treatment for uncomplicated cystitis (Hooton, 2012). Many recurrent UTIs are thought to arise from the ability of bacteria to attach to and invade the bladder mucosa, where they can form intracellular reservoirs protected from antibiotics and host defenses (Barber et al., 2013).

\section{Pathophysiology of Urinary Tract Infections}

Symptomatic UTI in a healthy female is a complex event. It is initiated by uropathogens that typically reside in the gut, followed by colonization of the urethra and subsequent migration of the pathogen to the bladder (Flores-Mireles et al., 2015). Female susceptibility to UTI is a function of basic anatomic factors as well as behavioral and psychologic factors that evolve over a lifetime. The short female urethra provides a bridge for invading pathogens and rapid ingress to the bladder. The proximity of the 
female urethra to bacterial reservoirs in the rectum and vagina also plays a crucial anatomic role (Dielubanza \& Schaeffer, 2011).

Pathogen adherence is a key event in initiating each step in UTI pathogenesis (Flores-Mireles et al., 2015). Appendages in the urethra such as flagella and pili allows colonization of the periurethral mucosa. In the bladder, uropathogens survive by binding directly to the bladder epithelium, which is composed of umbrella cells, intermediate cells, and basal cells. The consequences of complex host-pathogen interactions ultimately determine whether uropathogens are successful in colonization or elimination (FloresMireles et al, 2015).

Urinary tract infections are caused by both Gram-negative and Gram-positive bacteria, as well as by certain fungi. The most common causative agent for both uncomplicated and complicated UTI is uropathogenic Escherichia coli (UPEC) (FloresMireles et al., 2015). In females, E. coli causes $75 \%$ - 95\% of episodes of uncomplicated cystitis and pyelonephritis (Hooton, 2012). For the agents involved in uncomplicated UTIs, UPEC is followed in prevalence by Klebsiella pneumoniae, Staphylococcus saprophyticus, Enterococcus faecalis, group B Streptococcus (GBS), Proteus mirabilis, Pseudomonas aeruginosa, Staphylococcus aureus and Candida spp. (Flores-Mireles et al.). However, when E. faecalis and GBS are isolated from the voided urine in women with symptoms of uncomplicated cystitis, they often represent contamination of the voided specimen (Hooton).

\section{Risk Factors for Urinary Tract Infections}

Risk factors for uncomplicated cystitis include sexual intercourse, use of spermicides, a new sex partner (within the last year), a previous UTI, and a history of 
UTI in a first degree relative (Hooton, 2012). Women between the ages of 16-35 are 35 times more likely to contract an uncomplicated UTI and one-third of all women will have at least one UTI by age 24 (Schaeffer \& Schaeffer, 2007). Dielubanza and Schaefer (2011) found a dose-dependent relationship between sexual intercourse and the risk of UTI, findings which are corroborated by the spike in the first infections among young adult women around the time of first sexual activity. Dielubanza and Schaefer reviewed a prospective study of 769 sexually active young women and found that the relative risk of UTI after sexual intercourse on day 1,3 , and 5 of the previous days was $1.42,2.83$, and 5.68 respectively. Dielubanza and Schaefer concluded that the use of spermicide, either alone or in conjunction with a barrier contraceptive (diaphragm, cervical cap, or male condom), are associated with increased risk of UTI. Spermicides, especially those containing nonoxonol-9, alter vaginal flora, thereby facilitating periurethral colonization with uropathogens. Antimicrobial use in the previous month increases the risk of infection by the same mechanism (Dielubanza \& Schaefer). A genetic predisposition to recurrent UTI is suggested by the strong association between a history of UTI in one or more first-degree female relatives and in increased risk of recurrent cystitis (Hooton). This genetic predisposition is due to E coli binding receptivity that is not limited to the vaginal and urethral mucosa but also includes buccal mucosa, suggesting genetic differences in mucosal properties rather than in local milieu (Dielubanza \& Schaefer).

\section{Diagnosing Urinary Tract Infections and Diagnostic Testing}

Initial diagnosis of acute uncomplicated cystitis is typically based on patient medical history, taking into account past individual and family health issues, sexual activity, and current symptoms (Barber et al., 2013). During initial evaluation, it is 
important for the clinician to fully investigate the patient's subjective symptoms. Dysuria is a common complaint in many conditions such as urethritis and vaginitis, which requires pathogen isolation and pelvic exam. Cystitis is more likely when symptoms include: frequency, urgency, or hematuria; when the onset of symptoms is sudden or severe; and when vaginal irritation and discharge are not present (Hooton, 2012). The probability of cystitis is greater than $50 \%$ in women with any symptoms of UTI and greater than $90 \%$ in women who have dysuria and frequency without vaginal discharge and irritation (Hooton). Even with these statistics, clinicians often rely on readily available commercial point of care (POC) dip-stick tests, microscopic urinalysis, and culture and sensitivity tests to aid in their diagnosis of uncomplicated cystitis.

Accuracy of these tests is dependent on the quality of the urine sample with reliability directly related to the collection method employed (Dielubanza \& Schaeffer, 2011). The preferred collection method commonly used in the outpatient setting is the midstream clean catch voiding method, which is also the least reliable (Dielubanza \& Schaeffer). This method allows for collection without the aid of a skilled practitioner or the need for office appointment, creating convenience for patient and practitioner alike, but it is mired by frequent contamination (Dielubanza \& Schaeffer). Even when perineal cleansing is performed correctly, nearly one-third of these specimens can be contaminated (Bent \& Saint, 2002).

Assessment for pyuria and bacteriuria is often performed with POC analyzers that test for leukocyte esterase and nitrates (Hooton, 2012). Pyuria is the presence of pus in the urine caused by the host's inflammatory response which produces leukocyte esterase. Nitrate is the product of bacterial reduction of urine nitrate, and suggests the presence of 
gram-negative bacteria (Hooton). The dipstick test is the most accurate for predicting UTI when the presence of either leukocyte esterase or nitrite is considered a positive result, with a sensitivity of $75 \%$ and a specificity of $82 \%$ (Hooton). Women with symptoms suggestive of a UTI have demonstrated that the presence of nitrates is highly predictive of a positive culture (PV+ $=75 \%$ to $95 \%)$; dipstick showing more than trace leukocytes is nearly as predictable (PV+ $=65 \%$ to $85 \%)$; and the presence of both is almost conclusive (PV+ = 95\%) (Michels \& Sands, 2015). However, results of the dipstick test provide little useful information when the history is strongly suggestive of UTI, since even negative results for both tests do not reliably rule out infection in such case (Hooton). The patient history is the most useful tool because false negative results for leukocyte esterase can occur in the setting of early infection, when bacteriuria is present without hearty host inflammatory response. (Dielubanza \& Schaeffer, 2011). False-negative results for nitrite can occur in the setting of infection with gram-positive or other non-nitrite-reducing organisms. False-positive results in both tests can result from sample contamination (Dielubanza \& Schaeffer).

Microscopic examination is also routinely used in aiding the clinician to diagnose uncomplicated cystitis and is typically performed at a reference lab, after the clinician interviews the patient, performs POC testing and treats the patient based upon subjective symptoms (Bent \& Saint, 2002). The procedure can be vital in the diagnosis of cystitis with the presence of white blood cells and the absence of vaginal epithelial cells. However, the high incidence of contamination with the clean catch urine specimens and possibility for false-negative findings if bacterial colony count is lower than $10^{5}$ colony 
forming unit/milliliter (CFU/ml) are significant confounding factors (Dielubanza \& Schaeffer, 2011).

Although medical history and urinalysis are sufficient for the diagnosis of most cases of uncomplicated cystitis, the gold standard for diagnosis of acute cystitis includes a bacteriological urine culture with identification of the causative agent and antimicrobial susceptibility testing. Using fresh, midstream urine, clinical confirmation of an uncomplicated UTI is classically defined as $\geq 10^{5} \mathrm{CFU} / \mathrm{ml}$ of bacteria in urine samples (Barber et al., 2013). However, it is important to note that $20 \%$ to $40 \%$ of women presenting with cystitis will have low bacterial counts of $10^{2}$ to $10^{4} \mathrm{CFU} / \mathrm{ml}$ (Dielubanza \& Schaeffer, 2011). Consequently, as little as $10^{3} \mathrm{CFU} / \mathrm{ml}$ urine, in the presence of overt UTI symptoms, is now considered sufficient for diagnosis of acute cystitis (Schmiemann et al., 2010). A culture of "no growth" in a woman with UTI symptoms should be interpreted with caution since most clinical laboratories do not quantify bacteria below a threshold of $10^{4} \mathrm{CFU} / \mathrm{ml}$ in voided urine specimens (Hooton, 2012). Therefore, Hooton concluded that urine culture is indicated in all women with suspected pyelonephritis but is not necessary for the diagnosis of uncomplicated cystitis, given the reliability of the patient's history in establishing the diagnosis and the delayed availability of culture results (Hooton).

\section{Antimicrobial Resistance}

Given the rapid rate at which antimicrobial resistance patterns are evolving, staying abreast of local patterns requires engagement with the literature and vigilance in one's own practice. It is a good practice to review one's outpatient urine culture results on a monthly or quarterly basis for trends in resistance in the immediate community 
(Dielubanza \& Schaeffer, 2011). One of the most ominous issues on the horizon for bacterial infections, with UTIs being no exception, is the rise of antibiotic-resistant organisms. One especially troubling example is the heightened incidence of sequence type 131 (ST131) strains of uropathogenic E. coli (UPEC). These strains often exhibit high levels of resistance to multiple antibiotics and have undergone rapid intercontinental dispersal over the last decade (Peireno \& Pitout, 2010). ST131 strains are an increasingly common cause of community acquired UTIs, spurring efforts to better identify and treat these resilient pathogens (Peireno \& Pitout, 2010; Pitout, 2012). Factors driving the global spread of ST131 strains are incompletely understood, but likely the acquisition of antibiotic resistant genes, such as those encoding extended-spectrum $\beta$-lactamases (ESBLs), and the capacity to effectively utilize a broad range of metabolites (Peireno \& Pitout, 2010). These characteristics may give ST131 strains a competitive advantage within host environments, increasing the likelihood of their dissemination within and between individuals (Barber et al., 2013).

The microbial spectrum of uncomplicated cystitis consists mainly of E. coli (7595\%), with occasional other species of Enterobacteriaceae, such as P. mirabilis and $K$. pneumoniae, and S. saprophyticus (Gupta et al., 2011). Other gram-negative and gram positive species are rarely isolated in uncomplicated cystitis. Therefore, local antimicrobial susceptibility patterns of $E$. coli in particular should be considered in empirical antimicrobial selection for uncomplicated cystitis. Since the resistance patterns of $E$. coli strains causing uncomplicated cystitis varies considerably between regions and countries, a specific treatment recommendation may not be universally suitable for all regions and countries (Gupta et al.). 


\section{Prescribing Guidelines and Urinary Tract Infections}

Antimicrobial agents used for the treatment of UTI accounts for $15 \%$ of all outpatient prescriptions (Dielubanza \& Schaeffer, 2011). In 2010, a panel of international experts was convened by the Infectious Diseases Society of America (ISDA), in collaboration with the European Society for Microbiology and Infectious Diseases (ESCMID), to update the 1999 Uncomplicated Urinary Tract Infection Guidelines by the ISDA. Since 1999, antimicrobial resistance among uropathogens causing uncomplicated cystitis and pyelonephritis and appreciation of the importance of the ecological adverse effects of antimicrobial therapy (collateral damage) has increased (Gupta et al., 2011). Also, newer agents and different durations of therapy have been studied and clinical outcomes have increasingly been reported (Gupta et al.).

For the update, the expert panel completed a review and analysis of data published since 1998. Computerized literature searches of the PubMed data bases were performed. The searches of the English-language literature from 1998 - 2008, using the terms cystitis or pyelonephritis with MESH terms of "acute uncomplicated UTI," "women," and specific antimicrobials and or classes of antimicrobials. To be included, the study had to be an open-label or randomized, clinical trial of treatment of women with symptoms of acute uncomplicated cystitis or pyelonephritis. At least one follow-up visit assessing antimicrobial or clinical response was required. Studies including $>10 \%$ men or patients with complicated UTIs were excluded. Non-English language studies were excluded because they could not be reliably reviewed by panel members (Gupta et al., 2011). 
The following recommendations were advanced by the ISDA for the optimal treatment for acute uncomplicated cystitis (premenopausal, nonpregnant women with no known urological abnormalities or comorbidities) with consideration of resistance and collateral damage (ecological adverse effects of antimicrobial damage):

1. Nitrofurantoin monohydrate/macrocrystals (100 $\mathrm{mg}$ twice daily for 5 days) is an appropriate choice for therapy due to minimal resistance and propensity for collateral damage and efficacy comparable to 3 days of trimethoprimsulfamethoxazole.

2. Trimethoprim-sulfamethoxazole (160/800 mg [1 double strength tablet] twice daily for 3 days) is an appropriate choice for therapy, given its efficacy assessed in numerous clinical trials, if local resistance rates of uropathogen causing acute uncomplicated cystitis do not exceed $20 \%$ or if the infecting strain is known to be susceptible.

i. The threshold of $20 \%$ as the resistance prevalence at which the agent is no longer recommended for empirical treatment of acute cystitis is based on expert opinion derived from clinical, in vitro, and mathematical modeling guides.

ii. In some countries/regions trimethoprim (100 $\mathrm{mg}$ twice daily for 3 days) is the preferred agent and is considered equivalent to trimethoprimsulfamethoxazole on the basis of data presented in the original guidelines.

iii. Data are insufficient to make a recommendation for other cystitis antimicrobials as to what resistance prevalence should be used to preclude their use for empirical treatment of acute cystitis. 
3. Fosfomycin trometamol (3 grams in a single dose) is an appropriate choice for therapy where it is available due to minimal resistance and propensity for collateral damage, but it appears to have inferior efficacy compared with standard short-course regimens according to data submitted to US Food and Drug Administration.

4. Pivmecillinam (400 $\mathrm{mg}$ bid for 3-7 days) is an appropriate choice for therapy in regions where it is available (not licensed and/or available for use in North America) because of minimal resistance and propriety for collateral damage, but it may have inferior efficacy compared with other available therapies.

5. The fluoroquinolones ofloxacin, ciprofloxacin, and levofloxacin, are highly efficacious in 3 day regimens but have a propensity for collateral damage and should be reserved for important uses other than acute cystitis and thus should be considered alternative antimicrobials for acute cystitis.

6. $\beta$-Lactam agents, including amoxicillin-clavulanate, cefdinir, and cefpodoximeproxetil, in a 3-7 day regimens are appropriate choices for therapy when other recommended agents cannot be used. Other $\beta$-Lactam, such as cephalexin, are less well studied but may also be appropriate in certain settings. The $\beta$-Lactams generally have inferior efficacy and more adverse effects, compared with other UTI antimicrobials. For this reason, $\beta$-Lactams other than pivmecillinam should be used with caution for uncomplicated cystitis.

7. Amoxicillin or ampicillin should not be used for empirical treatment given the relatively poor efficacy as discussed in the 1999 guidelines and the very high prevalence of antimicrobial resistance to these agents worldwide. 
(Gupta et al., 2011).

\section{Remote Management Strategies and Urinary Tract Infections}

In 2001, Barry, Horner, Ebell, \& Enhorfner compared the outcomes of uncomplicated UTI in healthy women managed over the telephone with those managed in the office. They enrolled patients $(n=86)$ from five primary care offices and one urgent care clinic that called for an appointment with UTI symptoms. Non-pregnant women 18 years and older completed an interview to confirm eligibility when they called their primary care physician with a suspected uncomplicated UTI. They randomly assigned women calling with UTI symptoms to either treatment by telephone (telephone group) or office based care (control group). Thirty-six women were assigned to each group. The patients assigned to the telephone group (TG) were asked to go to the clinic, leave a urine sample, and pick up a seven-day course of antibiotics. The control group (CG) was given a same day appointment for a regular office visit with a clinician who was instructed to provide their usual management strategies (Barry et al.).

The authors developed a UTI rating score (0-50), which was determined by asking the patients to rate their severity of dysuria, urgency, frequency, function, and how they generally felt about their symptoms on a 10-point scale with 10 being the most severe. Both groups had similar UTI scores at baseline $(\mathrm{CG}=29.8, \mathrm{TG}=30.2)$. A nurse telephoned all women in both groups for follow up three and ten days after enrollment to assess patient status by reassessing their UTI score. There were no significant differences in the UTI scores on days three $(\mathrm{CG}=6.6, \mathrm{TG}=7)$ and ten $(\mathrm{CG}=3.0, \mathrm{TG}=1.7)$. Improvement in UTI scores from baseline were also similar on day three $(\mathrm{CG}=18.8$, $\mathrm{TG}=20.1)$ and day ten $(\mathrm{CG}=27.1, \mathrm{TG}=28.5)$ (p. 591). To determine an overall evaluation 
rating (OER) the women were asked about their overall satisfaction on a 5-point Likert scale $(\mathrm{CG}=$ very good, $\mathrm{TG}=$ excellent). They recorded start and stop times for all interviews to assist in estimating and comparing costs of care for each group. The nursing time for the day three follow up took an average of 5.6 minutes $(\mathrm{SD}=2.9)$ and 5.2 minutes $(\mathrm{SD}=2.0)$ on day ten (Barry et al.).

Of the 72 participants, five did not have culture results. Of the 67 remaining cultures, four $(6 \%)$ were contaminated, $20(29.8 \%)$ cultures were negative, and 43 (64.2\%) were positive. Out of the 43 positive cultures, 34 (79.1\%) grew out E. coli. There were minimal statistical differences between the CG and TG. The CG had $23(67.6 \%)$ positive cultures as compared to $20(60.6 \%)$ in the treatment group. The authors concluded that short term outcomes of managing suspected UTIs by telephone appears to be consistent with usual office care (Barry et al.).

In 2011 Blozik, Sommer-Meyer, Cerezo, and von Overbeck performed a study to evaluate the safety and effectiveness of the telemedical management of uncomplicated urinary tract infections (UUTI) including teleprescription of an antibiotic according to evidence-based guidelines in the Swiss Center for Telemedicine Medgate. Five hundred and twenty-six women between 18-65 years old were included. Participants were managed following evidence based protocol guidelines for the management of dysuria, issued by the German College of General Practitioners and Family Physicians (DEGAM). The participants included stated obvious symptoms (dysuria, frequency, and urgency) without diagnosis other than uncomplicated UTI, had no contraindication of antibiotic therapy, symptoms less than seven days and no relevant comorbidities according to a predefined list. Females with fever or chills, back or flank pain, vaginal discharge, 
pregnancy, UUTI recurrence within two weeks and treatment with antibiotics within the previous two weeks were excluded.

A three-day course of antibiotics was faxed to the participants' pharmacy of choice and were prescribed based upon DEGAM guidelines. The first line treatment was trimethoprim/sulfamethoxazole160/800 mg twice daily. Second line therapies included norfloxacine $400 \mathrm{mg}$ twice daily for three days or ciprofloxacine $250 \mathrm{mg}$ twice daily. Follow up was conducted three days after teleconsultation. Results were very similar to US based studies with $92 \%$ of participants reporting a reduction of UTI symptoms with $79 \%$ reporting complete symptom relief on day three. Although no significant levels were reported, Blozik et al. compared their data to literature on UUTI and concluded that telemedical management of UTI is as safe and effective as UUTI managed face-to-face with a high potential for saving a considerable number of consultations and healthcare dollars (Blozik et al., 2010).

In 2014, Grigoryan et al. searched PubMed and Cochrane data bases for English language studies published before July 21, 2014 with the objectives of defining the optimal approach for treating acute cystitis in young healthy women and defining the optimal approach for diagnosing acute cystitis in the outpatient setting. The initial review included 27 randomized clinical trials (RCT), six systematic reviews, and 11 observational studies focused on accurately diagnosing UTIs and appropriate treatment regimens. Due to increased resistance rates of trimethoprim-sulfamethoxazole, fluoroquinolones, and $\beta$-lactams, data published before 2000 was excluded along with studies that included antibiotics that were not available in the United States. Studies that only included pregnant women, children younger than twelve, adults older than sixty- 
five, or studies that included enrolled patients with factors that suggested complicated cystitis were also excluded.

Grigoryan et al. (2014) reviewed nine observational cohort studies, one systematic review, and one RCT on diagnosing UTIs and determined that uncomplicated acute cystitis in women can be diagnosed without an office visit or urine culture. Two key points were made in their review on the benefits of culture sparing strategies. First, urine cultures are not typically available to guide diagnosis or therapy during acute presentation and women who present to outpatient clinics with at least two symptoms of UTI (dysuria, urgency, or frequency) and the absence of vaginal discharge had a greater than $90 \%$ probability of having acute cystitis (Grigoryan et al.). Second, additional testing with a urine dipstick for leukocyte esterase would not further improve the likelihood of true infection, given the high pretest probability. Obtaining a urine sample either for dipstick testing or for culture in women with symptoms of acute cystitis was not associated with benefits in symptom scores or time to consultation compared with immediate empirical therapy. Thus, an office visit without a urine culture is an acceptable management strategy for acute cystitis (Grigoryan et al.).

In defining optimal antibiotic therapy for treating cystitis, Grigoryan et al. (2014) investigated and compared the efficacy of trimethoprim-sulfamethoxazole, nitrofurantoin, and fosfomycin. A total of 33 studies were included when defining the optimal approach for treating acute uncomplicated cystitis in the outpatient setting. When comparing trimethoprim-sulfamethoxazole with other agents in young women with acute uncomplicated cystitis, early clinical and bacterial cure rates were $85 \%-100 \%$ (Grigoryan et al., 2014). In the largest trial reviewed, overall clinical cure (30 days after 
therapy) was achieved in $79 \%$ of the trimethoprim-sulfamethoxazole group, and early clinical and microbiological cure rates were $90 \%$ and $91 \%$ respectively. It is helpful to know the local rate of trimethoprim-sulfamethoxazole resistance among community uropathogens because efficacy rates will differ based upon the prevalence of in vitro resistance. If the resistance prevalence is less than $20 \%$ and the local antibiogram or individual risk factors do not predict resistance, trimethoprim-sulfamethoxazole (160/800 mg twice daily for three days) is an appropriate choice for therapy (level of evidence A-I) (Grigoryan et al.).

Grigoryan et al. (2014) compared nitrofurantoin with other antimicrobial agents for uncomplicated cystitis in five RTCs and determined that nitrofurantoin monohydrate/macrocrystals (100 $\mathrm{mg}$ twice daily for 5-7 days) is also an appropriate choice for therapy because of its efficacy compared with three days of trimethoprimsulfamethoxazole and minimal resistance (level of evidence A-I). A five-day regimen can be considered in lieu of seven days on the basis of one RCT finding it comparable with three days of trimethoprim-sulfamethoxazole (level of evidence B-I) (Grigoryan et al.).

Grigoryan et al. (2014) compared the efficacy of a three-gram single dose of fosfomycin trometamol with other antimicrobial agents for uncomplicated cystitis in six RCTs. They noted that although the bacterial efficacy is lower than trimethoprimsulfamethoxazole, a single dose of fosfomycin three-gram dose is appropriate choice for uncomplicated cystitis (level of evidence A-I) (Grigoryan et al.).

Grigoryan et al. (2014) concluded that immediate antimicrobial therapy with trimethoprim-sulfamethazine, nitrofurantoin, or fosfomycin is indicated for acute cystitis in adult women. Increasing resistance rates among uropathogens have complicated 
treatment of acute cystitis, but telephone management without an office visit or culture is still an appropriate and efficient approach for most cases of uncomplicated cystitis. Individual assessment of risk factors for resistance and regimen tolerability is needed to choose the optimum empirical regimen (Grigoryan et al.).

\section{Remote Management Strategies: Self Diagnosis and Urinary Tract Infections}

In the right clinical setting, symptoms alone can identify patients with a high likelihood of UTI who are candidates for empirical therapy (Michels \& Sands, 2015). Women with an uncomplicated history who present with acute dysuria, urinary urgency or frequency, and no vaginal discharge can be treated for acute cystitis without other evaluation. Approximately $50 \%$ of patients that had a UTI found that the highest positive predictive value (PV+) of cystitis in women was self-diagnosis of cystitis (86\%), followed by the absence of vaginal discharge (82\%), presence of hematuria (75\%), and urinary frequency $(73 \%)$. The combination of dysuria and urinary frequency without vaginal discharge or irritation yielded a very high likelihood of UTI (positive likelihood ratio $[\mathrm{LR}+]=24.6)($ Michels \& Sands).

Women with a history of UTI can often identify symptoms indicting onset of subsequent UTI. In self-diagnosis or patient initiated therapy, women with a previous UTI are often provided with an antimicrobial regimen to keep at home, or a prescription refill, with instructions to initiate therapy at the onset of symptoms (Grigoryan et al., 2014). An existing patient-clinician relationship and education of the patient to seek care if there are new symptoms (vaginal discharge/irritation, or back pain/fever), a late or missed menstrual period, or a new sex partner are essential for the implementation of this 
approach. This strategy allows women to address their symptoms rapidly and efficiently, while avoiding missed diagnosis of STD or pyelonephritis (Grigoryan et al.).

In summary, the evidence in the reviewed literature review supports the proposal for a retrospective chart review to determine the accuracy of remote management of uncomplicated cystitis. Acute uncomplicated cystitis is a common condition that can often be successfully diagnosed and treated without a urine culture. Culture sparing strategies include remote management, patient self-diagnosis, and office visits without culture. This approach is effective in reducing costs while improving patient satisfaction, with no increase in adverse outcomes.

Several research studies were identified to support the proposal. Woman with dysuria and frequency, no risk factors for complicated infection, and no vaginal discharge had a $90 \%$ probability of UTI (Hooton, 2012). Women presenting to outpatient clinics with at least two symptoms of UTI (dysuria, urgency, or frequency) and the absence of vaginal discharge had a greater than $90 \%$ probability of having acute cystitis; thus treatment based on symptoms was advocated (Bent et al, 2002). Knottnerus et al. (2013), in his study of 196 symptomatic women, found that $79 \%$ of patients with "considerable" dysuria, suspicion of UTI, and absence of vaginal discharge had also a UTI. Little et al. (2010) also concluded that additional testing with a urine dipstick for leukocyte esterase would not further improve the likelihood of true infection, given the high pretest probability. The authors concluded that obtaining a urine sample either for dipstick testing or for culture in women with symptoms of acute cystitis was not associated with benefits in symptom scores or time to reconstitution compared with immediate empirical therapy (Little et al.). 
Next, the theoretical framework used to guide this project will be described. 


\section{Theoretical Framework}

Steven's ACE Star Model of Knowledge Transformation (Stevens, 2015) was utilized as a guide for this quality project to offer a simple yet comprehensive approach to translate evidence into practice. The ACE Star Model of Knowledge Transformation was developed in 2004 and highlights barriers encountered when moving evidence into practice and designates solutions grounded in evidence based practice (EBP) (Stevens). The model explains how various stages of knowledge transformation reduce the volume of scientific literature and provide forms of knowledge that can be directly incorporated in care and decision making. The ACE Star Model emphasizes crucial steps to convert one form of knowledge to the next and incorporate best research evidence with clinical expertise and patient preferences thereby achieving EBP (Stevens). The ACE Star Model provides a framework for systematically putting evidence based practice into operation. The model is comprised of five major stages: knowledge discovery, evidence summary, translation into practice recommendations, integration into practice, and evaluation (Figure 1). 


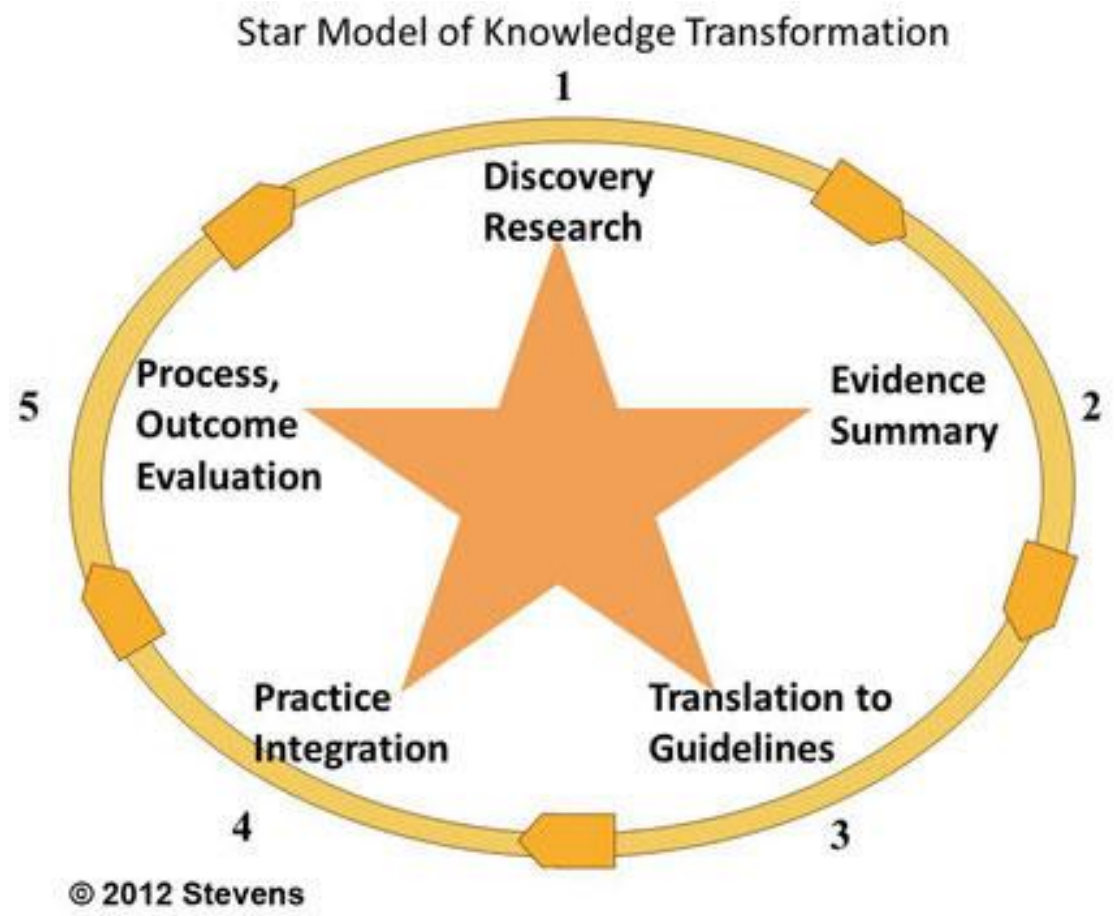

Figure 1

The model depicts various forms of knowledge in a relative sequence, as research evidence is moved through several cycles, combined with other knowledge and integrated into practice (Stevens, 2015). This same sequence of knowledge transformation can be applied to this quality improvement project. The feasibility of remote management of uncomplicated UTI has been outlined in the literature review.

For stages 1 and 2, discovery research and evidence summary, evidence of "knowledge generating" has been obtained through thorough primary research studies to support the concept of remote management of UTIs. Evidence summary has been supported in the literature review with evidence from systematic reviews and randomized control trials. The third stage, translation to guidelines, transforms the evidence presented during stages 1 and 2 into practice recommendations for remote management of uncomplicated UTIs. During this stage, the outcomes of the proposed project will determine the feasibility of remote management by determining how often subjective 
symptoms of uncomplicated urinary tract infections were associated with treatment sensitive to subsequent urine cultures in an urgent care setting.

The fourth stage, practice integration, allows the clinician to implement remote management of uncomplicated UTIs based upon the knowledge gained from evidence based research performed during the previous stages in the model. The fifth stage, process outcome evaluation, allows for the clinician to evaluate the implementation of remote management of uncomplicated UTIs on patient outcomes, patient and provider satisfaction, efficacy, cost, and health status.

Next the methodology will be discussed. 


\section{Method}

\section{Purpose}

The purpose of this quality improvement project was to determine how often subjective symptoms of uncomplicated urinary tract infections were associated with treatment sensitive to subsequent urine cultures in an urgent care setting.

\section{Design}

The design for this project was a non-experimental retrospective chart review. Site

The quality project was conducted at Concentra Urgent Care in Warwick, RI. Concentra treats approximately 50 urgent care patients daily for non-life threatening illnesses and injuries. Based upon the clinic population profile, at least three patients present daily to the clinic with uncomplicated UTI symptoms.

\section{Sample}

The sample for this project included medical records of premenopausal (ages 1845) non-pregnant females treated for UTI between January 1, 2016 and July 1, 2016.

The inclusion criteria included subjective symptoms of dysuria, urinary urgency, and urinary frequency. Exclusion criteria were pregnancy, presence of symptoms consistent with sexually transmitted infection, vaginal discharge, upper abdominal pain, flank pain, back pain, history of kidney stones, risk for pyelonephritis (fever [temperature $>38^{\circ} \mathrm{C}$ ], chills, flank pain, costovertebral-angle tenderness, and nausea or vomiting, with or without symptoms of cystitis) (Hooton, 2012) and history of recurrent UTIs as defined by two uncomplicated infections in a six month time period or three infections within a year (Dielubanza \& Schaeffer, 2011) or urinary symptoms that persist or recur within a week or two of treatment for uncomplicated cystitis (Hooton, 2012). 


\section{Measurement}

The author developed a data collection (Appendix A) tool, based on the literature and clinical experience, to collect and organize the data. The data collection tool was created in Microsoft Excel and include the participant number, age of the participant, and various subjective data including dysuria, urinary frequency, urinary urgency, and suprapubic pain, prescribed antibiotic, culture pathogen result, and was the treatment sensitive to the pathogen. The subjective data were collected in both the medical assistant and provider notes section in the participant's chart. Culture pathogen results were reviewed on the antibiogram from the reference lab and then compared to the prescribed antibiotic to determine if appropriate antibiotics were prescribed.

\section{Procedures}

All data were collected by the student researcher after written approval was granted by Concentra's Center Operations Director, Medical Director, and the Concentra legal team. The project was determined to be exempt by the Rhode Island College IRB.

Charts that meet the inclusion criteria were selected from the All-Scripts Electronic Medical Records system. Each record was assigned chronological numbers, and only that number was recorded; no PHI were collected or recorded. Data collection took place in a private area to maintain privacy. All data were stored on a password protected thumb drive and stored in a locked cabinet at Concentra Warwick facility with access limited to the author only.

\section{Data Analysis}

Basic descriptive statistics were utilized to analyze the data including means, ranges, and percentages. 


\section{Results}

A total of 84 charts were reviewed and 37 met the criteria of uncomplicated cystitis. Symptom data were retrieved from both the medical assistant and provider notes and is illustrated on Table 1 on the following page. The average age of the subjects at time of visit was 28.28 years with a range of $18-45$ years. All of the participants presented with subjective symptoms of dysuria, urinary frequency and urinary urgency. Sixteen participants (43.24\%) reported symptoms of suprapubic pain and another sixteen (43.24\%) did not report it. In the remaining five participants (13.51\%), symptoms of suprapubic pain were not documented.

All of the subjects except one $(n=36 ; 97.29 \%)$ were prescribed an antibiotic at time of visit. Thirty-four samples $(91.89 \%)$ were sent to an external reference lab for further culture and sensitivity testing, while three urine samples $(8.10 \%)$ were not. Four cultures $(11.76 \%)$ tested negative for bacterial growth, with $30(88.23 \%)$ testing positive for bacterial growth based on the antibiogram results. Of the 30 positive samples, 29 $(85.29 \%)$ were treated with an appropriate antibiotic at the time of visit based on the antibiogram results received from the reference lab. One sample $(2.94 \%)$ was identified with an intermediate bacterial resistance and was later changed to another antibiotic when culture results were reviewed.

Next, the summary and conclusions will be presented. 
Table 1.

Data Collection Table $(N=37)$

\begin{tabular}{|c|c|c|c|c|c|c|c|c|}
\hline Participant & Age & Dysuria & Frequency & Urgency & $\begin{array}{c}\text { Suprapubic } \\
\text { pain }\end{array}$ & $\begin{array}{c}\text { Prescribed } \\
\text { Antibiotic }\end{array}$ & \begin{tabular}{|c|} 
Culture \\
Result
\end{tabular} & $\begin{array}{l}\text { Pathogen } \\
\text { Sensitive }\end{array}$ \\
\hline 1 & 34 & $\mathrm{Y}$ & $\mathrm{Y}$ & $\mathrm{Y}$ & $\mathrm{Y}$ & A & $(+)$ & $\mathrm{Y}$ \\
\hline 2 & 26 & $\mathrm{Y}$ & $\mathrm{Y}$ & $\mathrm{Y}$ & ND & $C$ & $(+)$ & $\mathrm{Y}$ \\
\hline 3 & 43 & $\mathrm{Y}$ & $\mathrm{Y}$ & $\mathrm{Y}$ & $\mathrm{Y}$ & $A$ & $(+)$ & $\mathrm{Y}$ \\
\hline 4 & 25 & $\mathrm{Y}$ & $\mathrm{Y}$ & $\mathrm{Y}$ & $\mathrm{N}$ & $\mathrm{B}$ & $(+)$ & $\mathrm{Y}$ \\
\hline 5 & 35 & $\mathrm{Y}$ & $\mathrm{Y}$ & $\mathrm{Y}$ & $\mathrm{Y}$ & A & $(+)$ & $\mathrm{Y}$ \\
\hline 6 & 28 & $\mathrm{Y}$ & $\mathrm{Y}$ & $\mathrm{Y}$ & $\mathrm{N}$ & C & $(-)$ & $N / A$ \\
\hline 7 & 21 & $\mathrm{Y}$ & $\mathrm{Y}$ & $\mathrm{Y}$ & ND & $A$ & $(+)$ & $\mathrm{Y}$ \\
\hline 8 & 42 & $\mathrm{Y}$ & $\mathrm{Y}$ & $\mathrm{Y}$ & $\mathrm{Y}$ & $\mathrm{C}$ & $(+)$ & $\mathrm{Y}$ \\
\hline 9 & 27 & $\mathrm{Y}$ & $\mathrm{Y}$ & $\mathrm{Y}$ & $\mathrm{N}$ & $\mathrm{D}$ & $(+)$ & $\mathrm{Y}$ \\
\hline 10 & 19 & $\mathrm{Y}$ & $\mathrm{Y}$ & $\mathrm{Y}$ & $\mathrm{N}$ & $A$ & $(+)$ & $\mathrm{Y}$ \\
\hline 11 & 38 & $\mathrm{Y}$ & $\mathrm{Y}$ & $\mathrm{Y}$ & $\mathrm{Y}$ & $\mathrm{A}$ & $(+)$ & $\mathrm{Y}$ \\
\hline 12 & 24 & $\mathrm{Y}$ & $\mathrm{Y}$ & $\mathrm{Y}$ & $\mathrm{Y}$ & $\mathrm{B}$ & $(+)$ & $\mathrm{Y}$ \\
\hline 13 & 21 & $\mathrm{Y}$ & $\mathrm{Y}$ & $\mathrm{Y}$ & $N$ & B & $(+)$ & $\mathrm{Y}$ \\
\hline 14 & 21 & $\mathrm{Y}$ & $\mathrm{Y}$ & $\mathrm{Y}$ & ND & $\mathrm{B}$ & $(-)$ & N/A \\
\hline 15 & 45 & $\mathrm{Y}$ & $\mathrm{Y}$ & $\mathrm{Y}$ & $\mathrm{Y}$ & A & $(+)$ & $\mathrm{Y}$ \\
\hline 16 & 32 & $\mathrm{Y}$ & $\mathrm{Y}$ & $\mathrm{Y}$ & ND & A & $(+)$ & $\mathrm{Y}$ \\
\hline 17 & 23 & $\mathrm{Y}$ & $\mathrm{Y}$ & $\mathrm{Y}$ & ND & A & $(+)$ & $\mathrm{Y}$ \\
\hline 18 & 35 & $\mathrm{Y}$ & $\mathrm{Y}$ & $\mathrm{Y}$ & $\mathrm{N}$ & A & $(+)$ & $\mathrm{Y}$ \\
\hline 19 & 28 & $\mathrm{Y}$ & $\mathrm{Y}$ & $\mathrm{Y}$ & $\mathrm{N}$ & A & $(+)$ & $Y$ \\
\hline 20 & 21 & $Y$ & $Y$ & $\mathrm{Y}$ & $\mathrm{N}$ & A & $(+)$ & $Y$ \\
\hline 21 & 28 & $\mathrm{Y}$ & $\mathrm{Y}$ & $\mathrm{Y}$ & $\mathrm{N}$ & C & $(-)$ & N/A \\
\hline 22 & 19 & $\mathrm{Y}$ & $\mathrm{Y}$ & $Y$ & $\mathrm{~N}$ & $\mathrm{~B}$ & $(+)$ & $\mathrm{Y}$ \\
\hline 23 & 25 & $Y$ & $Y$ & $\mathrm{Y}$ & $\mathrm{Y}$ & $\mathrm{B}$ & $(+)$ & $Y$ \\
\hline 24 & 22 & $\mathrm{Y}$ & $\mathrm{Y}$ & $\mathrm{Y}$ & $\mathrm{Y}$ & C & $(+)$ & $\mathrm{Y}$ \\
\hline 25 & 25 & $\mathrm{Y}$ & $\mathrm{Y}$ & $Y$ & $\mathrm{Y}$ & $\mathrm{B}$ & $(+)$ & $\mathrm{Y}$ \\
\hline 26 & 27 & $\mathrm{Y}$ & $\mathrm{Y}$ & $\mathrm{Y}$ & $\mathrm{Y}$ & B & (?) & $U$ \\
\hline 27 & 32 & $\mathrm{Y}$ & $\mathrm{Y}$ & $\mathrm{Y}$ & $\mathrm{N}$ & C & $(-)$ & $\mathrm{N} / \mathrm{A}$ \\
\hline 28 & 18 & $Y$ & $Y$ & $Y$ & $\mathrm{~N}$ & $A$ & $(+)$ & $Y$ \\
\hline 29 & 35 & $Y$ & $\mathrm{Y}$ & $\mathrm{Y}$ & $\mathrm{N}$ & A & $(+/-)$ & $\mathrm{I}$ \\
\hline 30 & 22 & $\mathrm{Y}$ & $\mathrm{Y}$ & $\mathrm{Y}$ & $\mathrm{Y}$ & A & $(+)$ & $\mathrm{Y}$ \\
\hline 31 & 31 & $\mathrm{Y}$ & $\mathrm{Y}$ & $\mathrm{Y}$ & $\mathrm{N}$ & C & $(+)$ & $\mathrm{Y}$ \\
\hline 31 & 28 & $\mathrm{Y}$ & $\mathrm{Y}$ & $\mathrm{Y}$ & $\mathrm{Y}$ & C & $(+)$ & $\mathrm{Y}$ \\
\hline 33 & 45 & $Y$ & $\mathrm{Y}$ & $\mathrm{Y}$ & $\mathrm{Y}$ & A & $(+)$ & $Y$ \\
\hline 34 & 20 & $Y$ & $\mathrm{Y}$ & $\mathrm{Y}$ & $Y$ & A & $(+)$ & $Y$ \\
\hline 35 & 26 & $\mathrm{Y}$ & $\mathrm{Y}$ & $\mathrm{Y}$ & $\mathrm{Y}$ & B & $(+)$ & $\mathrm{Y}$ \\
\hline 36 & 20 & $\mathrm{Y}$ & $\mathrm{Y}$ & $\mathrm{Y}$ & $\mathrm{N}$ & $\mathrm{B}$ & (?) & $u$ \\
\hline 37 & 35 & $\mathrm{Y}$ & $\mathrm{Y}$ & $\mathrm{Y}$ & $\mathrm{N}$ & C & (?) & $u$ \\
\hline
\end{tabular}
$\mathrm{Y}=\mathrm{Yes}$
$\mathrm{N}=\mathrm{No}$
$(+)=$ Positive
$(-)$ Negative

$\mathrm{ND}=$ Suprapubic pain was not documented at visit

(?) = no culture was sent at visit

$\mathrm{A}=$ Nitrofurantoin monohydrate/macrocrystals

$\mathrm{B}=$ Trimethoprim-sulfamethoxazole

$\mathrm{C}=$ Fluoroquinolones (ofloxacin, ciprofloxacin, and levofloxacin)

$\mathrm{D}=$ Antibiotic not prescribed at visit

N/A = negative culture result, therefore not applicable 


\section{Summary and Conclusions}

Urinary tract infection (UTI) is the chief complaint in eight million clinic and emergency department visits, leading to approximately 100,000 hospital admissions each year (Dielubanza \& Schaeffer, 2011). The evaluation and treatment of community acquired UTI commands a significant portion of health care resources. Currently, the societal costs of these infections, including health care costs and time missed from work, are approximately $\$ 3.5$ billion per year in the United States alone (Flores-Mireles, et al., 2015). Traditional UTI management requires the patient to seek treatment by face-to-face consultation with a medical provider, receive point of care testing and have urine sample sent to a reference lab for culture and sensitivity. Research has supported that uncomplicated UTIs can be appropriately managed remotely with similar results as office consultations (Hooton, 2012).

The purpose of this quality improvement project was to determine how often subjective symptoms of uncomplicated urinary tract infections were associated with treatment sensitive to subsequent urine cultures in an urgent care setting. The theory used to guide this this quality improvement model was Steven's ACE Star Model of Knowledge Transformation (2015). The data collection took place at Concentra Urgent Care in Warwick, RI. Concentra treats approximately 50 urgent care patients daily for non-life threatening illnesses and injuries.

The inclusion criteria included subjective symptoms of dysuria, urinary urgency and urinary frequency. Exclusion criteria were pregnancy, presence of symptoms consistent with sexually transmitted infection, vaginal discharge, upper abdominal pain, flank pain, back pain, history of kidney stones, risk for pyelonephritis (fever [temperature $>38^{\circ} \mathrm{C}$, chills, flank pain, costovertebral-angle tenderness, and nausea or vomiting, with 
or without symptoms of cystitis (Hooton, 2012), and history of recurrent UTIs as defined by two uncomplicated infections in a six month time period or three infections within a year (Dielubanza \& Schaeffer, 2011) or urinary symptoms that persist or recur within a week or two of treatment for uncomplicated cystitis (Hooton).

Eighty-four medical records of premenopausal (ages 18-45) non-pregnant females treated for UTI between January 1, 2016 and July 1, 2016 were identified during a retrospective chart review. Thirty-seven participants met the inclusion criteria for uncomplicated cystitis. The average age of the subjects at time of visit was 28.28 years. All of the participants presented with subjective symptoms of dysuria, urinary frequency and urinary urgency. Sixteen participants (43.24\%) reported symptoms of suprapubic pain and another $16(43.24 \%)$ did not. In the remaining five participants $(13.51 \%)$, symptoms of suprapubic pain were not documented.

All of the subjects except one $(n=36 ; 97.29 \%)$ were prescribed an antibiotic at time of visit. Thirty-four urine samples $(91.89 \%)$ were sent for culture and sensitivity testing, while three urine samples $(8.10 \%)$ were not. Four cultures $(11.76 \%)$ tested negative for bacterial growth, with $30(88.23 \%)$ testing positive for bacterial growth. Of the 30 positive samples, $29(85.29 \%)$ were treated with an appropriate antibiotic based on the antibiogram results received from the reference lab at the time of visit. One sample (2.94\%) was identified with an intermediate bacterial resistance and was later changed to another antibiotic when culture results were reviewed.

Several limitations were identified in this research project. The research was performed at one urgent care clinic. Incomplete and inconsistent documentation of subjective symptoms of cystitis limited data collection. Not all the charts reviewed had 
documented subjective symptoms of dysuria, frequency, and urgency which limited the sample size to 36 . The sample size could have been significantly larger if all the subjective symptoms were documented appropriately based upon the literature review recommendations. Also, different providers document subjective symptoms differently. Finally, there was not an attempt to evaluate potential treatment differences in ethnic populations.

In summary, this study investigated how often subjective symptoms of uncomplicated urinary tract infections were associated with treatment sensitive to subsequent urine cultures in an urgent care setting. Findings were consistent with research performed by Barry et al. (2001) and Hooton (2012). Barry et al. concluded that $64 \%$ of patients were appropriately treated remotely based upon subjective symptoms. Hooton concluded that $90 \%$ of patients could be treated with appropriate antibiotics based upon local resistance patterns and their subjective symptoms. This research validated that $85.29 \%$ of patients could have been treated appropriately based solely on their subjective symptoms of uncomplicated cystitis.

Next, recommendations and implications for APRNs will be discussed. 


\section{Recommendations and Implications for Advanced Nursing Practice}

One in three women will develop a UTI requiring antibiotic treatment by age 24 and 50\% of women will experience at least one UTI during their lifetime (Dielubanza \& Schaeffer, 2011). Research and evidence-based practice for remote management strategies for uncomplicated cystitis had been introduced to clinicians via research publications for several decades. Yet, trends in practice have not evolved with the evidence provided. The costs of healthcare are rising and the current societal costs for treating these infections, including health care costs and time missed from work, are approximately $\$ 3.5$ billion per year in the United States alone (Flores-Mireles et al., 2015). All clinicians must look at strategies that will reduce health care costs while providing competent care to their patients and balance their responsibilities to patients and the institutions where they are employed. Advanced practice registered nurses (APRN) can act as change agents by developing policies, educating staff on current research results and healthcare trends and educating peers and patients on the benefits of remote management strategies. Advanced practice registered nurses must focus on strategies that will reduce health care costs while providing evidence-based practice for proper UTI management.

This quality improvement project investigated the outcomes of urine cultures sent to a reference lab for sensitivity testing to determine if the patients could have been treated remotely based upon their subjective symptoms with the same accuracy. Thirtyseven subjects met the inclusion criteria for this project. All participants presented with subjective symptoms of dysuria, urinary frequency and urinary urgency. As evidenced by the retrospective chart review, all subjects except one $(n=36 ; 97.29 \%)$ were prescribed an antibiotic before the sample was sent for culture. The findings from this sample 
represented an $85.29 \%$ accuracy rate. Based upon the results, patients can be treated remotely based upon their subjective symptoms which supports that this strategy can work in clinical practice. The remote management strategy would have yielded the same results without added healthcare costs.

Advanced practice nurses can utilize available innovative health care technologies such as telemedicine and patient portals to appropriately manage uncomplicated urinary tract infections remotely. Telemedicine is becoming more prevalent in health care models and can be implemented to treat uncomplicated cystitis. Patient portals and the use of electronic medical records help the provider in several ways. Clinicians can easily review medical records at the touch of a button to inquire about recent office visits, recent antibiotic treatments, complications of previous treatments, drug allergies and comorbidities that may affect appropriate treatment regimens. These innovative technologies can help reduce costs, decrease burden on healthcare scheduling and decrease time restraints on patients.

There must be a knowledgeable relationship between the patient and provider. This model might not work in an urgent care practice but could be implemented in a primary care setting where the provider often has a closer relationship with their patients. Clinician and patient education is key for this concept to work. The provider must take the time to determine the reliability of the information provided and decide if remote management is appropriate based upon the patient's symptoms and habits. The clinician must able to navigate appropriate questions that distinguish between uncomplicated cystitis and other medical complications of complicated cystitis, pyelonephritis, vaginal infections, yeast infection, pregnancy and sexually transmitted infections. The provider 
and patient must have an open, honest relationship about the patient's medical history, sexual habits and risk factors. If the patient is at risk for complications, an office visit with appropriate examinations must be performed.

Advanced Practice Registered Nurses can work as leaders in developing policies for remote management strategies and take on the role of policy development for these changes. Institutions and independent medical practices rely on the knowledge of policy development which APRNs receive as part of their educational curriculum. As leaders, APRNs must be informed about healthcare policies at local, institutional and national levels. Advanced practice nurses must also be up to date on institutional policies and protocols for remote management strategies. Some institutions may not have policies that address telephone consultations and remote management strategies. On a national level, APRNs must be knowledgeable about recommendations based upon the current ISDA uncomplicated urinary tract infection guidelines.

Advanced Practice Registered Nurses receive extensive education and training in order to become independent and competent clinicians, utilizing critical thinking during physical examination and health assessment. Advanced Practice Registered Nurses use extensive assessment techniques in clinical settings to assess the whole person during face-to-face interviews. However, telemedicine and remote management strategies are not part of current education curriculums. These topics can easily be implemented in educational programs. Advanced Practice Registered Nurses can attend lectures, seminars and training classes as part of their continuing education credits. Institutions and independent medical facilities can develop training modules, standing orders and 
implement standard operating procedures to address remote management strategies of uncomplicated UTIs.

Advanced practice nurses promote best practice for health promotion and illness prevention based on several ethical principles. With beneficence, APRNs have an obligation to prevent and remove harms while weighing possible benefits against possible risks of an action. When interviewing the patient remotely, the APRN must still take time to discuss side effects of treatments and educate the patients on preventative measures along with STI education. The APRN must also practice autonomy. Autonomous behavior includes presenting all treatment options to the patient while explaining risks in terms that the patient understands, ensuring that the patient understands the risks and agrees to the plan of care. The patient on the other end of the telephone must also be capable of making autonomous decisions and be in the right state of mind to give informed consent using logical reasoning.

Advanced Practice Registered Nurses must remain up to date on current evidenced based practice and research for treatment options for uncomplicated UTIs. The APRN must be up to date on treatments that affect individual risk factors such as drug allergies and treatment outcomes based upon local resistance prevalence which affects efficacy or empirical treatment regimens. Although much research has been performed on antibiotic prescribing and antibiotic resistance, there is a limited research being performed on remote management strategies. Further research is needed in this area, including research that incorporates cultural considerations.

In summary, APRNs are leaders in research, policy development, policy implementation and patient education. The results of this research support the statement 
that uncomplicated UTIs can be treated remotely with the same efficacy as those treated by traditional office management. Healthcare dollars can be significantly reduced by following this strategy while also improving patient satisfaction. 


\section{References}

Barber, A. E., Norton, J. P., Spivak, A. M., \& Mulvey, M. A. (2013). Urinary tract infections: current and emerging management strategies. Clinical Infectious Disease, 57(5), 719-724.

Barry, H. C., Hickner, J., Ebell, M., \& Ettenhofer, T. (2001). A randomized controlled trial of telephone management of suspected urinary tract infections in women. Journal of Family Practice, 50(7), 589-594.

Bent, S. \& Saint, S. (2002). The optimal use of diagnostic testing in women with acute uncomplicated cystitis. The American Journal of Medicine, 113(1), 20-28.

Blozik, E., Sommer-Meyer, C., Cerezo, M., \& von Overbeck (2011). Effectiveness and safety of telemedical management in uncomplicated urinary tract infections. Journal of Telemedicine \& Telecare, 17(2), 78-82.

Colgan, R., \& Williams M. (2011). Diagnosis and treatment of acute uncomplicated cystitis. American Family Physician, 84(7), 771-776.

Dielubanza, E. J. \& Schaeffer, A. J. (2011). Urinary tract infections in women. Medical Clinics of North America. 95(1), 27-41.

Flores-Mireles, A. L., Walker, J. N., Caparon, M., \& Hultgren, S. J., (2015). Urinary tract infections: epidemiology, mechanisms of infection and treatment options. Nature Reviews Microbiology, 13(5), 269-284.

Foxman, B. (2002). Epidemiology of urinary tract infections: incidence, morbidity, and economic costs. The American Journal of Medicine. 113(1), 5-13.

Foxman, B. (2014). Urinary tract infection syndromes; occurrences, recurrence, bacteriology, risk factors, and disease burden. Infectious Disease Clinics of North America, 28(1), 1-13. 
Griebling, T., L. (2007). Urinary tract infection in women. Urologic Diseases in America. Department of Health and Human Services, Public Health Service, National Institutes of Health, National Institute of Diabetes and Digestive and Kidney Diseases. Washington, D.C.: GPO; 2007. NIH publication 07-5512:587619.

Grigoryan, L., Trautner, B. W., \& Gupta, K. (2014). Diagnosis and management of urinary tract infections in the outpatient setting: a review. JAMA, 312(16), 16771684.

Gupta, K., Hooton, T. M., Naber, K. G., Wullt, B., Colgan, R., Miller, L. G., ... \& Soper, D. E. (2011). International clinical practice guidelines for the treatment of acute uncomplicated cystitis and pyelonephritis in women: a 2010 update by the Infectious Diseases Society of America and European Society for Microbiology and infectious Disease. Clinical Infectious Disease. 52(5), e103-e120.

Gupta, K., Hooton, T. M., \& Stamm, W., E. (2001). Increasing antimicrobial resistance and the management of uncomplicated community-acquired urinary tract infections. Annals of Internal Medicine. 135(1), 41-50.

Hooton, T.M. (2012). Uncomplicated urinary tract infection. New England Journal of Medicine, 366(11), 1028-1037.

Kattan, J.N., \& Gordon, S. (2013). Acute uncomplicated urinary tract infections. Retrieved from: http://www.clevelandclinicmeded.com/medicalpubs/diseasemanagement/infectiou s-disease/urinary-tract-infection/ 
Knottnerus, B. J., Geerlings, S. E., van Charante, E. P. M., \& ter Riet, G. (2013). Toward a simple diagnostic index for acute uncomplicated urinary tract infections. The Annals of Family Medicine, 115(5), 442-451.

Little, P., Moore, M. V., Turner, S., Rumsby, K., Warner, G., Lowes, J. A., ... \& Turner, D. (2010). Effectiveness of five different approaches in management of urinary tract infection; randomised controlled trial. BMJ, 340, c199.

Michels, T. C., \& Sands, J. E. (2015). Dysuria: Evaluation and Differential Diagnosis in Adults. American Family Physicians. 92(9).

National Institutes of Health. (2013). How many women are affected or at risk for UTIs and UI? Retrieved from: https://www.nichd.nih.gov/health/topics/urinary/conditioninfo/Pages/affected.asp $\mathrm{x} \# \mathrm{f} 3$

Peirano, G., \& Pitout, J. D. (2010). Molecular epidemiology of Escherichia coli producing CTX-M $\beta$-lactamases: the worldwide emergence of clone ST131 025: H4. International Journal of Antimicrobial Agents, 35(4), 316-321.

Pitout, J. D. (2012). Extraintestinal pathogenic Escherichia coli: an update on antimicrobial resistance, laboratory diagnosis and treatment. Expert Review of Anti-Infective Therapy, 10(10), 1165-1176.

Schaeffer, A. J., \& Schaefer, E. M. (2007). Infections of the urinary tract. In Wein, A. J., Kavoussi, L. R. (9 $\left.{ }^{\text {th }}\right)$, Cambel-Walsh Urology. (223-302). Philadelphia: Saunders Elsevier. 
Schmiemann, G., Kniehl, E., Gebhardt, K., Matejczyk, M. M., Hummers-Pradier, E. (2010). The diagnosis of urinary tract infection: a systematic review. Deutsches Ärzteblatt International, 107(46), 824-825.

Stevens, K. (2015). ACE Star Model of Evidence Based Practice: Knowledge Transformation. Retrieved from: http://www.aahs.org/aamcnursing/?page_id=745 


\section{Appendix A}

\begin{tabular}{|c|c|c|c|c|c|c|c|c|}
\hline Participant\# & Age & $\begin{array}{l}\text { Dysuria } \\
\text { Y/N }\end{array}$ & $\begin{array}{c}\text { Frequency } \\
\mathrm{Y} / \mathrm{N}\end{array}$ & $\begin{array}{l}\text { Urgency } \\
\mathrm{Y} / \mathrm{N}\end{array}$ & $\begin{array}{c}\text { Suprapubic } \\
\text { pain Y/N }\end{array}$ & $\begin{array}{c}\text { Prescribed Antibiotic } \\
\text { (see legend) }\end{array}$ & $\begin{array}{c}\text { Culture } \\
\text { Pathogen Result }\end{array}$ & $\begin{array}{c}\text { Antibiotic Sensitive to } \\
\text { Pathogen } \mathrm{Y} / \mathrm{N}\end{array}$ \\
\hline \multicolumn{9}{|l|}{1} \\
\hline \multicolumn{9}{|l|}{2} \\
\hline \multicolumn{9}{|l|}{3} \\
\hline \multicolumn{9}{|l|}{4} \\
\hline \multicolumn{9}{|l|}{5} \\
\hline \multicolumn{9}{|l|}{6} \\
\hline \multicolumn{9}{|l|}{7} \\
\hline \multicolumn{9}{|l|}{8} \\
\hline \multicolumn{9}{|l|}{9} \\
\hline \multicolumn{9}{|l|}{10} \\
\hline \multicolumn{9}{|l|}{11} \\
\hline \multicolumn{9}{|l|}{12} \\
\hline \multicolumn{9}{|l|}{13} \\
\hline \multicolumn{9}{|l|}{14} \\
\hline \multicolumn{9}{|l|}{15} \\
\hline \multicolumn{9}{|l|}{16} \\
\hline \multicolumn{9}{|l|}{17} \\
\hline \multicolumn{9}{|l|}{18} \\
\hline \multicolumn{9}{|l|}{19} \\
\hline \multicolumn{9}{|l|}{20} \\
\hline \multicolumn{9}{|l|}{21} \\
\hline \multicolumn{9}{|l|}{22} \\
\hline \multicolumn{9}{|l|}{23} \\
\hline \multicolumn{9}{|l|}{24} \\
\hline 25 & & & & & & & & \\
\hline
\end{tabular}

Prescribed antibiotic:

A. Nitrofurantoin monohydrate/macrocrystals

B. Trimethoprim-sulfamethoxazole

C. Fosfomycin trometamol

D. Fluoroquinolones (ofloxacin, ciprofloxacin, and levofloxacin)

E. $\beta$-Lactam agents, including amoxicillin-clavulanate, cefdinir, and cefpodoximeproxetil

F. Amoxicillin or ampicillin 